\title{
Transient analysis of cycle lengths in cyclic polling systems
}

\author{
P. Vis ${ }^{\text {a,b,* }}$, R. Bekker ${ }^{a}$, R.D. van der Mei ${ }^{\text {b,a }}$ \\ a VU University Amsterdam, Amsterdam, Netherlands \\ ${ }^{\mathrm{b}}$ Centre for Mathematics and Computer Science, Amsterdam, Netherlands
}

\section{A R T I C L E I N F O}

\section{Article history:}

Available online 6 July 2015

\section{Keywords:}

Polling models

Transient analysis

Cycle times

Correlations

Waiting times

\begin{abstract}
A B S T R A C T
We consider cyclic polling models with gated or globally gated service, and study the transient behavior of all cycle lengths. Our aim is to analyze the dependency structure between the different cycles, as this is an intrinsic property making polling models challenging to analyze. Moreover, the cycle structure is related to the output of a polling model and the current analysis may be useful to study networks of polling models. In addition, transient performance is of great interest in systems where disruptions or breakdowns may occur, leading to excessive cycle lengths. The time to recover from such events is a primary performance measure. For the analysis we assume that the distribution of the first cycle (globally gated) or $N$ residence times (gated), where $N$ is the number of queues, is known and that the arrivals are Poisson. The joint Laplace-Stieltjes transform (LST) of all $x$ subsequent cycles (globally gated) or all $x>N$ subsequent residence times (gated) is expressed in terms of the LST of the first cycle. From this joint LST, we derive first and second moments and correlation coefficients between different cycles. Finally, a heavy-tailed first cycle length or the heavy-traffic regime provides additional insights into the time-dependent behavior.
\end{abstract}

(C) 2015 Elsevier B.V. All rights reserved.

\section{Introduction}

Polling systems are multi-queue systems in which a single server visits the queues in some order to serve the customers waiting at the queues, typically incurring some amount of switch-over time to proceed from one queue to the next. Polling models find a wide variety of applications in which processing power (e.g., CPU, bandwidth, manpower) is shared among different types of users. Typical application areas of polling models are computer-communication systems, logistics, flexible manufacturing systems, production systems and maintenance systems; the reader is referred to [1] for an extensive overview of the applicability of polling models. Over the past few decades the performance analysis of polling models has received much attention in the literature. We refer to Vishnevskii and Semenova [2] for an excellent overview of the available results on polling models.

In this paper, we study cyclic polling models with gated or globally gated service, and focus on the transient behavior of the successive cycle times. Our goal is to gain an understanding in the dependency structure between the different cycles. This study is motivated by our interest in systems where disruptions or breakdowns may occur, often leading to excessive cycle lengths. In this context, we are interested in the following questions:

1. If the system encounters an excessively long cycle time (e.g., due to a disruption or a breakdown), then how will that influence the durations of the subsequent cycle times? What is the time needed to recover from excessive cycle times?

\footnotetext{
* Corresponding author at: VU University Amsterdam, Amsterdam, Netherlands.

E-mail address: petra.vis@vu.nl (P. Vis).
} 
2. What is the dependency structure between various residence and cycle times? More specifically, what is the correlation between the successive cycle (and residence) times?

A primary motivation for the second question is that the dependency structure makes polling models challenging to analyze. Insights into the dependency between cycles and residence times might pave the way for approximation methods. For instance, for polling models in tandem, the output of some queues may feed into another queue. The output of a specific queue in a polling system is essentially driven by an on-off source with dependent on and off times ('on' representing visit times and 'off' representing intervisit times). Similar relations have also motivated the study of some vacation models, see e.g. [3-5]. Finally, we note that waiting-time and queue-length distributions can be expressed in terms of the marginal cycle-time distribution for polling models with (globally) gated and exhaustive service.

In this paper, we assume that the distribution of the first cycle (in case of globally gated service) or $N$ residence times (in case of gated service), where $N$ is the number of queues, is known and that the arrivals are Poisson. Using this, we show how the joint Laplace-Stieltjes transform (LST) of all $x$ subsequent cycles (globally gated) can be expressed in terms of the LST of the first cycle. Moreover, for the case of gated service we show how all $x>N$ subsequent residence times can be expressed in terms of the LST of the first cycle. From these joint LST's, we derive the first two moments and correlation coefficients between different cycles. Lastly, we analyze a heavy-tailed first cycle length, due to disruptions or breakdown, or the heavy-traffic regime to provide new fundamental insights into the time-dependent behavior.

The remainder of this paper is organized as follows. In Section 2 the models are described and the method and goals of the paper are outlined. In Section 3 we study the case of globally-gated service, whereas we study the case of gated service in Section 4. Both sections contain asymptotic results, such as heavy-tailed initial cycle lengths and heavy traffic, and numerical illustrations.

\section{Model, method and goals}

\subsection{Model description}

We consider a system of $N \geq 2$ infinite-buffer queues, $Q_{1}, \ldots, Q_{N}$, and a single server that visits and serves the queues in cyclic order. Customers arrive at $Q_{i}$ according to a Poisson process $\left\{N_{i}(t), t \in \mathbb{R}\right\}$ with rate $\lambda_{i}$. These customers are referred to as type-i customers. The total arrival rate is denoted by $\Lambda=\sum_{i=1}^{N} \lambda_{i}$. The service time of a type-i customer is a random variable $B_{i}$, with LST $B_{i}^{*}(\cdot)$, and $k$ th moment $\mathbb{E}\left[B_{i}^{k}\right], k=1,2, \ldots$, when it is finite. The $k$ th moment of the service time of an arbitrary customer is denoted by $\mathbb{E}\left[B^{k}\right]=\sum_{i=1}^{N} \lambda_{i} \mathbb{E}\left[B_{i}^{k}\right] / \Lambda, k=1,2, \ldots$ The load offered to $Q_{i}$ is $\rho_{i}=\lambda_{i} \mathbb{E}\left[B_{i}\right]$ and the total load offered to the system is equal to $\rho=\sum_{i=1}^{N} \rho_{i}$. The switch-over time required by the server to proceed from $Q_{i}$ to $Q_{i+1}$ is a random variable $S_{i}$ with mean $\mathbb{E}\left[S_{i}\right]$ and LST $S_{i}^{*}(\cdot)$. Let $S=\sum_{i=1}^{N} S_{i}$, with LST $S^{*}(\cdot)$, denote the total switch-over time in a cycle. We define $\delta_{i}(s):=\lambda_{i}\left(1-B_{i}^{*}(s)\right)$ and let $\mathbf{e}_{i}$ be a unit vector with 1 in the $i$ th position and 0 in the other positions.

We consider the gated and globally gated service disciplines. When the service discipline is gated, a gate at $Q_{i}$ closes when the server arrives at $Q_{i}$. Every customer standing in front of the gate is served, while customers arriving at $Q_{i}$ during service of $Q_{i}$ must wait for the next cycle, this holds for all $i=1, \ldots, N$. When the service discipline is globally gated, a gate closes at all queues when the server arrives at $Q_{1}$. During the following cycle, every customer standing in front of the gate is served.

\subsection{Method and goals}

Throughout we assume that the distribution of the length of the first cycle is known. For the gated service discipline, this requires that the joint distribution of the first $N$ residence times is known, where a residence time is a visit time plus the subsequent switch-over time. When the probabilistic behavior of the first cycle is known, the next residence time can be expressed in terms of the first cycle, as it consists of a visit time to serve all the work that arrived at the queue during the first cycle plus the switch-over time. For globally gated, this is true for every queue, as the gate closes at the start of a cycle. For gated, the length of a visit time is always determined by the work that arrived at the corresponding queue during the last $N$ residence times. It can be seen that the second cycle is completely determined in terms of the first cycle. Consequently, the third cycle can be expressed in terms of the second cycle and so also in terms of the first cycle. As a result, every cycle can recursively be expressed in terms of the first cycle. We use this fact to derive the joint LST of $x$ consecutive cycles or residence times in terms of the LST of the first cycle.

Let us first consider the globally gated case. Our goal is to determine the joint LST of $x$ consecutive cycle times, denoted by $\gamma_{x}(\mathbf{z})$. The vector $\mathbf{z}$ of length $x$ contains the variables $z_{1}, \ldots, z_{x}$, corresponding to cycles $1, \ldots, x$, with the LST of the first cycle, $\gamma_{1}(\mathbf{z})$, assumed to be given. Choosing the $z_{i}$ in specific ways, enables us to calculate all kinds of useful performance measures. For example, when $z_{i}=z$ for all $i \in J \subseteq\{1, \ldots, x\}$ and 0 otherwise, we obtain the LST of the sum of cycles of set $J$. Such a choice is especially convenient to calculate moments, which are then obtained by differentiating with respect to $z$ and taking $z=0$.

Also, the covariance between cycle 1 and cycle $x$ can be calculated using the following property of the covariance: if $X_{1}$ and $X_{2}$ are random variables, then $\operatorname{Var}\left(X_{1}+X_{2}\right)=\operatorname{Var}\left(X_{1}\right)+\operatorname{Var}\left(X_{2}\right)+2 \operatorname{Cov}\left(X_{1}, X_{2}\right)$, with the variance of a random variable 
$X$ being $\operatorname{Var}(X)=\mathbb{E}\left[X^{2}\right]-\mathbb{E}[X]^{2}$. To calculate the covariance between cycle $x$ and cycle $y(y \geq x)$, we define 3 vectors of length $y$ : $\mathbf{a}=z\left(\mathbf{e}_{x}+\mathbf{e}_{y}\right), \mathbf{b}=z \mathbf{e}_{x}$ and $\mathbf{c}=z \mathbf{e}_{y}$. Let $C_{i}$ denote the $i$ th cycle. The first vector is then used to calculate the variance of $C_{x}+C_{y}$, the other two vectors are used for the variance of $C_{x}$ and $C_{y}$, respectively. The covariance is then given by

$$
\operatorname{Cov}\left(C_{x}, C_{y}\right)=\frac{1}{2}\left(\operatorname{Var}\left(C_{x}+C_{y}\right)-\operatorname{Var}\left(C_{x}\right)-\operatorname{Var}\left(C_{y}\right)\right) .
$$

If, due to some external event or disaster, the first cycle is very long, we can easily derive the duration of the effect by calculating the duration of subsequent cycles, until it converges to the expected duration of $\mathbb{E}[S] /(1-\rho)$, if $\rho<1$. In addition, the recursive relations allow us to derive exact asymptotic transient results for some limiting regimes, such as heavy traffic and heavy-tailed initial cycle times.

For gated, the same methods can be applied, for residence times instead of cycle times. By choosing $N$ consecutive $z_{i}$ equal to $z$, it is also possible to look at cycles. Note that the state description for gated in terms of residence times can also be applied to the globally gated case to obtain information about the residence times.

\section{Globally gated service discipline}

In this section we consider the joint LST of $x$ cycles for a globally gated service discipline. This result is used to derive various moments and asymptotic properties. We suppose that the distribution of the first cycle, $C_{1}$, is known, and that its LST is given by $\gamma_{1}(z):=\mathbb{E}\left[e^{-z C_{1}}\right]$. Because of the globally gated service discipline, the length of a cycle determines the number of customers that are served during the next cycle. The number of customers that are served during a cycle plus the switch-over times together determine the length of that cycle. As such every cycle length can be expressed in the length of the previous cycle, and it is possible to express every cycle length recursively in terms of the first cycle. If we want to give the joint LST of $C_{1}$ and $C_{2}$, in terms of the LST of $C_{1}$, we can first condition on the value of $C_{1}$ and then integrate over the density of $C_{1}$, as follows:

$$
\begin{aligned}
\mathbb{E}\left[e^{-z_{1} c_{1}-z_{2} C_{2}}\right] & =\int_{c_{1}=0}^{\infty} \mathbb{E}\left[e^{-z_{1} c_{1}-z_{2} C_{2}}\right] \mathrm{d} \mathbb{P}\left(C_{1} \leq c_{1}\right) \\
& =\int_{c_{1}=0}^{\infty} \mathbb{E}\left[e^{-z_{1} c_{1}-\sum_{k=1}^{N} \lambda_{k}\left(1-B_{k}^{*}\left(z_{2}\right)\right) c_{1}}\right] \mathbb{E}\left[e^{-z_{2} S}\right] \mathrm{d} \mathbb{P}\left(C_{1} \leq c_{1}\right) \\
& =C_{1}^{*}\left(z_{1}+\sum_{k=1}^{N} \delta_{k}\left(z_{2}\right)\right) S^{*}\left(z_{2}\right),
\end{aligned}
$$

where $C_{1}^{*}(\cdot)$ is the LST of $C_{1}$, which we defined as $\gamma_{1}(\cdot)$. More generally, we can write the joint LST of the first $x$ cycles, denoted by $\gamma_{x}(\cdot)$, in terms of the LST of the first cycle. Here, $\mathbf{z}$ is a vector of length $x$, with $z_{i}$ corresponding to cycle $i$.

Theorem 1. The joint transform of the $x$ cycle lengths is given by

$$
\gamma_{x}(\mathbf{z})=\mathbb{E}\left[e^{-\sum_{i=1}^{x} z_{i} C_{i}}\right]=\gamma_{1}\left(z_{1}+\zeta_{1}^{(x)}(\mathbf{z})\right) \prod_{j=1}^{x-1} S^{*}\left(z_{j+1}+\zeta_{j+1}^{(x-j)}(\mathbf{z})\right),
$$

with

$$
\begin{aligned}
& \zeta_{n}^{(1)}(\mathbf{z})=0 \\
& \zeta_{n}^{(i)}(\mathbf{z})=\sum_{k=1}^{N} \delta_{k}\left(z_{n+1}+\zeta_{n+1}^{(i-1)}(\mathbf{z})\right) .
\end{aligned}
$$

Note that $\zeta_{n}^{(i)}$ can be interpreted as the amount of work that arrived during the $n$th cycle and recursively contains the amount of work that arrives during the next $(i-1)$ cycles, i.e., in some sense it defines a descendant set, see also Remark 1 . In the proof of Theorem 1, we use the following lemma (with the proof deferred to Appendix A).

Lemma 1. For $i=2,3, \ldots$ and $n=1,2, \ldots$, we have

$$
\zeta_{n}^{(i)}\left(\mathbf{z}^{\prime}\right)=\zeta_{n}^{(i+1)}(\mathbf{z})
$$

with $\mathbf{z}^{\prime}=\mathbf{z}+\zeta_{n+i-1}^{(2)}(\mathbf{z}) \cdot \mathbf{e}_{n+i-1}$. 
Proof of Theorem 1. We use induction to prove the theorem. It evidently holds for $x=1$. Now assume that Eq. (3) holds for $k \leq x$. Taking $k=x+1$ gives

$$
\begin{aligned}
\gamma_{(x+1)}(\mathbf{z}) & =\gamma_{x}\left(z_{1}, z_{2}, \ldots, z_{x}+\sum_{k=1}^{N} \delta_{k}\left(z_{x+1}\right)\right) S^{*}\left(z_{x+1}\right) \\
& =\gamma_{x}\left(z_{1}, z_{2}, \ldots, z_{x}+\zeta_{x}^{(2)}(\mathbf{z})\right) S^{*}\left(z_{x+1}+\zeta_{x+1}^{(1)}(\mathbf{z})\right) \\
& =\gamma_{1}\left(z_{1}+\zeta_{1}^{(x)}\left(\mathbf{z}^{\prime}\right)\right) \prod_{j=1}^{x-1} S^{*}\left(z_{j+1}+\zeta_{j+1}^{(x-j)}\left(\mathbf{z}^{\prime}\right)\right) S^{*}\left(z_{x+1}+\zeta_{x+1}^{(1)}(\mathbf{z})\right) \\
& =\gamma_{1}\left(z_{1}+\zeta_{1}^{(x+1)}(\mathbf{z})\right) \prod_{j=1}^{x-1} S^{*}\left(z_{j+1}+\zeta_{j+1}^{(x-j+1)}(\mathbf{z})\right) S^{*}\left(z_{x+1}+\zeta_{x+1}^{(1)}(\mathbf{z})\right) \\
& =\gamma_{1}\left(z_{1}+\zeta_{1}^{(x+1)}(\mathbf{z})\right) \prod_{j=1}^{x} S^{*}\left(z_{j+1}+\zeta_{j+1}^{(x-j+1)}(\mathbf{z})\right) .
\end{aligned}
$$

For the first equality, we use a reasoning similar to Eq. (2), then we rewrite the result using (4). For the third equality, the induction hypotheses is used. For the fourth equality, we use Lemma 1, completing the proof.

Remark 1 (Link with the Descendant Set Approach). The expression in Theorem 1 can be explained along the lines of the Descendant Set Approach (DSA) [6]. The DSA considers original customers (originators) and non-original customers, where an original customer arrives during a switch-over period, and a non-original customer arrives during the service of another customer; The children of a customer $T$ arrive during the service of $T$. The descendant set of $T$ is recursively defined to consist of $T$, its children and the descendants of its children. In our case, the first cycle and the $x-1$ switch-over times can be interpreted as originators and the recursive definition of $\zeta_{n}^{(i)}(\mathbf{z})$ represents the descendant sets. We note that in this case all quantities are expressed in time (or amount of work), while in the DSA this is typically in terms of the number of customers.

By choosing specific values for $z_{i}, i=1, \ldots, x$, many performance measures can be determined, see also Section 2.2. Below, we focus on the first two moments and the correlation coefficient. Here we assume that $z_{i}$ is either $z$ or 0 (depending on whether cycle $i$ is included). Let $J \subseteq\{1,2, \ldots, x\}$ be the set of cycles that is included, i.e., $z_{i}=z$ if and only if $i \in J$. Taking the derivative of $\gamma_{x}$, with respect to $z_{x}$, taking $z=0$ and multiplying by -1 , gives the expected length of cycle $x$. The derivative of $\gamma_{x}$, with respect to $z$, then becomes

$$
\begin{aligned}
\frac{d}{d z} \gamma_{x}(\mathbf{z})= & \frac{d}{d z} \gamma_{1}\left(z_{1}+\zeta_{1}^{(x)}(\mathbf{z})\right)\left(\mathbb{1}_{\{1 \in J\}}+\frac{d}{d z} \zeta_{1}^{(x)}(\mathbf{z})\right) \prod_{j=1}^{x-1} S^{*}\left(z_{j+1}+\zeta_{j+1}^{(x-j)}(\mathbf{z})\right) \\
& +\gamma_{1}\left(z_{1}+\zeta_{1}^{(x)}(\mathbf{z})\right) \sum_{j=1}^{x-1} \frac{d}{d z} S^{*}\left(z_{j+1}+\zeta_{j+1}^{(x-j)}(\mathbf{z})\right)\left(\mathbb{1}_{\{j+1 \in J\}}+\frac{d}{d z} \zeta_{j+1}^{(x-j)}(\mathbf{z})\right) \prod_{k \neq j}^{x-1} S^{*}\left(z_{k+1}+\zeta_{k+1}^{(x-k)}(\mathbf{z})\right),
\end{aligned}
$$

with $\frac{d}{d z} \zeta_{n}^{(i)}(\mathbf{z})$ recursively defined as

$$
\frac{d}{d z} \zeta_{n}^{(i)}(\mathbf{z})=\sum_{k=1}^{N} \frac{d}{d z} \delta_{k}\left(z_{n+1}+\zeta_{n+1}^{(i-1)}(\mathbf{z})\right)\left(\mathbb{1}_{\{n+1 \in J\}}+\frac{d}{d z} \zeta_{n+1}^{(i-1)}(\mathbf{z})\right), \quad i \geq 2,
$$

and $\frac{d}{d z} \zeta_{n}^{(i)}(\mathbf{z})=0$ if $i=1$. Here $\frac{d}{d z} \delta_{k}(z)=-\lambda_{k} B_{k}^{*}(z)$. Taking $z=0$ gives $\left.\frac{d}{d z} \delta_{k}(z)\right|_{z=0}=\rho_{k}$, and filling in $z=0$ in Eq. (7) gives the next property:

Property 1. We have, for $i=2,3, \ldots$ and $n=1,2, \ldots$,

$$
\left.\frac{d}{d z} \zeta_{n}^{(i)}(\mathbf{z})\right|_{z=0}=\sum_{k=1}^{i-1} \rho^{k} \mathbb{1}_{\{n+k \in J\}} .
$$

Proof. Eq. (8) can be proved by induction. First, take $i=2$, yielding

$$
\frac{d}{d z} \zeta_{n}^{(2)}(\mathbf{z})=\sum_{k=1}^{N} \frac{d}{d z} \delta_{k}\left(z_{n+1}\right) \mathbb{1}_{\{n+1 \in J\}} .
$$


If $z=0$, this equals $\sum_{k=1}^{N} \rho_{k} \mathbb{1}_{\{n+1 \in J\}}=\rho \mathbb{1}_{\{n+1 \in J\}}$, which agrees with (8). Now assume (8) is true for all $k \leq i$. Taking $k=i+1$ gives

$$
\frac{d}{d z} \zeta_{n}^{(i+1)}(\mathbf{z})=\sum_{k=1}^{N} \frac{d}{d z} \delta_{k}\left(z_{n+1}+\zeta_{n+1}^{(i)}(\mathbf{z})\right)\left(\mathbb{1}_{\{n+1 \in\}\}}+\frac{d}{d z} \zeta_{n+1}^{(i)}(\mathbf{z})\right)
$$

according to (7), and for $z=0$, we obtain

$$
\begin{aligned}
\left.\frac{d}{d z} \zeta_{n}^{(i+1)}(\mathbf{z})\right|_{z=0} & =\sum_{k=1}^{N} \rho_{k}\left(\mathbb{1}_{\{n+1 \in J\}}+\sum_{l=1}^{i-1} \rho^{l} \mathbb{1}_{\{n+1+l \in J\}}\right) \\
& =\rho \mathbb{1}_{\{n+1 \in J\}}+\sum_{l=1}^{i-1} \rho^{l+1} \mathbb{1}_{\{n+1+l \in J\}} \\
& =\rho \mathbb{1}_{\{n+1 \in J\}}+\sum_{l=2}^{i} \rho^{l} \mathbb{1}_{\{n+l \in J\}}=\sum_{k=1}^{i} \rho^{l} \mathbb{1}_{\{n+l \in J\}},
\end{aligned}
$$

where the first equality holds due to the induction hypotheses, completing the proof.

Combining the above yields the following proposition, for the expectation of the summation of the lengths of cycles $i \in J$.

Proposition 1 (First Moments). The expected total length of all cycles in set $J$ is

$$
\mathbb{E}\left[\sum_{j \in J} C_{j}\right]=\mathbb{E}\left[\gamma_{1}\right] \sum_{k=0}^{x-1} \rho^{k} \mathbb{1}_{\{k+1 \in J\}}+\mathbb{E}[S] \sum_{j=1}^{x-1} \sum_{l=0}^{x-j-1} \rho^{l} \mathbb{1}_{\{j+l+1 \in J\}},
$$

where $\mathbb{E}\left[\gamma_{1}\right]$ is the expected length of the initial cycle.

To calculate the variances of specific cycles, or covariances between two cycles, we also need the second moments. To this end, we take the derivative of Eq. (6), resulting in Eq. (19) in Appendix B. The derivative of (7) is given by Eq. (20) in Appendix B. Taking $z=0$ leads to the following property:

Property 2. We have, for $i=2,3, \ldots$ and $n=1,2, \ldots$,

$$
\left.\frac{d^{2}}{d z^{2}} \zeta_{n}^{(i)}(\mathbf{z})\right|_{z=0}=-\Lambda \mathbb{E}\left[B^{2}\right] \sum_{k=0}^{i-2} \rho^{k}\left(\sum_{j=0}^{i-k-2} \rho^{j} \mathbb{1}_{\{n+j+k+1 \in J\}}\right)^{2} .
$$

The proof is by induction and is similar to the proof of Property 1 .

Proposition 2 (Second Moments). Let $\mathbb{E}\left[\gamma_{1}^{2}\right]$ be the second moment of the initial cycle. The second moment of the total cycle length of all cycles in $\mathrm{J}$ is

$$
\begin{aligned}
\mathbb{E}\left[\left(\sum_{j \in J} C_{j}\right)^{2}\right]= & \mathbb{E}\left[\gamma_{1}^{2}\right]\left(\sum_{k=0}^{x-1} \rho^{k} \mathbb{1}_{\{k+1 \in J\}}\right)^{2}+\mathbb{E}\left[\gamma_{1}\right]\left(2 \mathbb{E}[S]\left(\sum_{k=0}^{x-1} \rho^{k} \mathbb{1}_{\{k+1 \in J\}}\right)\left(\sum_{j=1}^{x-1} \sum_{k=0}^{x-j-1} \rho^{k} \mathbb{1}_{\{j+1+k \in J\}}\right)\right. \\
& \left.+\Lambda \mathbb{E}\left[B^{2}\right] \sum_{k=0}^{x-2} \rho^{k}\left(\sum_{j=0}^{x-k-2} \rho^{j} \mathbb{1}_{\{j+k+2 \in J\}}\right)^{2}\right) \\
& +\mathbb{E}[S] \Lambda \mathbb{E}\left[B^{2}\right] \sum_{j=1}^{x-1} \sum_{k=0}^{x-j-2} \rho^{k}\left(\sum_{l=0}^{x-j-k-2} \rho^{l} \mathbb{1}_{\{j+l+k+2 \in J\}}\right)^{2}+\mathbb{E}\left[S^{2}\right] \sum_{j=1}^{x-1}\left(\sum_{k=0}^{x-j-1} \rho^{k} \mathbb{1}_{\{j+k+1 \in J\}}\right)^{2} \\
& +\mathbb{E}[S]^{2}\left(\left(\sum_{j=1}^{x-1} \sum_{k=0}^{x-j-1} \rho^{k} \mathbb{1}_{\{j+k+1 \in J\}}\right)^{2}-\sum_{j=1}^{x-1}\left(\sum_{k=0}^{x-j-1} \rho^{k} \mathbb{1}_{\{j+k+1 \in J\}}\right)^{2} .\right.
\end{aligned}
$$

Using Eq. (1) and the moments derived above, we can calculate the covariance between cycles 1 and $x$. This gives

$$
\begin{aligned}
\operatorname{Cov}\left(C_{1}, C_{x}\right) & =\frac{1}{2}\left(\mathbb{E}\left[\gamma_{x}(\mathbf{a})^{2}\right]-\mathbb{E}\left[\gamma_{x}(\mathbf{a})\right]^{2}-\left(\mathbb{E}\left[\gamma_{x}(\mathbf{b})^{2}\right]-\mathbb{E}\left[\gamma_{x}(\mathbf{b})\right]^{2}\right)-\left(\mathbb{E}\left[\gamma_{x}(\mathbf{c})^{2}\right]-\mathbb{E}\left[\gamma_{x}(\mathbf{c})\right]^{2}\right)\right) \\
& =\left(\mathbb{E}\left[\gamma_{1}^{2}\right]-\mathbb{E}\left[\gamma_{1}\right]^{2}\right) \rho^{x-1},
\end{aligned}
$$


where the second equality is obtained by filling in Eqs. (9) and (10). Interestingly, we see that the covariance between the first cycle and cycle $x$ only depends on the variance of the first cycle and the load of the complete system.

Remark 2 (Steady-State Distribution). In polling models, it is common to relate the length of cycle 2 to the length of the first cycle. Assuming the system to be in steady state, this provides an equation for the LST of the steady-state cycle length, see e.g. [7]. In fact Eq. (27) of [7] is equivalent to (2) if we take $z_{1}=0$. A similar recursive scheme can also be found in [8]. The distinguishing feature is that $[8,7]$ focus on steady-state results, whereas our aim is to derive transient performance.

Alternatively, putting $z_{x}=z$ and $z_{i}$ equal to zero for $i<x$, gives information about cycle $x$. Taking $x \rightarrow \infty$, also gives information about a cycle in steady state. This limit in Eq. (8) gives exactly $\mathbb{E}[S] /(1-\rho)$, if $\rho<1$. Doing the same with Eq. (10), gives exactly the second moment of a steady state cycle. This shows that our expressions are in line with known results (see again $[8,7]$ ).

\subsection{Asymptotic properties}

From Theorem 1 and Eq. (11) we may derive more explicit results for the different asymptotic regimes, such as light and heavy traffic and large switch-over times. Moreover, we consider the case in which the first cycle has a heavy-tailed distribution, representing the situation that this cycle is affected by an external event.

Light and heavy traffic. In heavy traffic, when $\rho \uparrow 1$, we directly obtain that the coefficient of correlation between cycles 1 and $x$ tends to 1 , see (11). Hence, the distribution of $C_{x}$ is identical to the distribution of $C_{1}$ in heavy traffic.

For light traffic, we observe from Eq. (11) that when the load tends to zero, the covariance between the first cycle and any other cycle is equal to zero, which also holds for the correlation coefficient. For approximations based on light and heavy traffic interpolations, the derivative of the light-traffic regime with respect to the load is useful. Remarkable is that the derivative of the covariance is zero for cycles $x=3,4, \ldots$, but it is strictly positive for the second cycle.

Large switch-over times. Another common asymptotic regime is the situation of large switch-over times, see [9]. Let the switch-over times be deterministic with length $r$, i.e. $S^{*}(z)=e^{-r z}$. We scale the cycle times by dividing by $r$ and let $r \rightarrow \infty$. This gives

$$
\begin{aligned}
\lim _{r \rightarrow \infty} \gamma_{x}(\mathbf{z} / r) & =\lim _{r \rightarrow \infty} \gamma_{1}\left(z_{1} / r+\zeta_{1}^{(x)}(\mathbf{z} / r)\right) \prod_{j=1}^{x-1} e^{-\left(z_{j+1} / r+\zeta_{j+1}^{(x-j)}(\mathbf{z} / r)\right) r} \\
& =\prod_{j=1}^{x-1} e^{-\left(\sum_{k=0}^{x-j-1} \rho^{k} z_{j+k+1}\right)}
\end{aligned}
$$

implying that the scaled cycle lengths become deterministic and are simple expressions in terms of the system load $\rho$.

Heavy-tailed initial cycle length. Let us assume that the first cycle is regularly varying of index $-v$ (denoted as $C_{1} \in \mathcal{R}_{-v}$; and with $m<v<m+1$ ), whereas the service and switch-over times have a lighter tail. This situation is of interest in cases where a disaster or external events leads to a long cycle. Of primary interest is how this initial cycle affects future cycle lengths.

More specifically, we assume that $\mathbb{P}\left(C_{1}>y\right) \sim L(y) y^{-v}, v>1$, with $L(y)$ some slowly varying function. We use the notation $f(y) \sim g(y)$ to indicate that $f(y) / g(y) \rightarrow 1$ as $y \rightarrow \infty$. A function $L(\cdot)$ is called slowly varying if $L(\eta y) \sim L(y)$, for all $\eta>1$. For the service and switch-over times, we assume the following:

Assumption A. $\mathbb{P}\left(B_{i}>y\right)=o\left(\mathbb{P}\left(C_{1}>y\right)\right)$ and $\mathbb{P}\left(S_{i}>y\right)=o\left(\mathbb{P}\left(C_{1}>y\right)\right)(i=1, \ldots, N)$.

Proposition 3. Suppose that $C_{1} \in \mathcal{R}_{-v}$ and Assumption A is satisfied. Then,

$$
\mathbb{P}\left(C_{n}>y\right) \sim \mathbb{P}\left(C_{1}>\frac{y}{\rho^{n-1}}\right) .
$$

The result of Proposition 3 can be intuitively explained and is related to the fact that rare events tend to occur due to a single most probable cause in systems with heavy-tailed characteristics. More precisely, the most likely way for a large $n$th cycle time is a large initial cycle time, whereas the system shows average behavior otherwise. As traffic arrives at rate $\rho$, the amount of traffic arriving during the first cycle is about $\rho C_{1}$; this means cycle 1 needs to be larger than $y / \rho$ for cycle 2 to be at least of length $y$.

The proof of the above proposition relies on the relation between the asymptotic behavior of regularly varying distributions and the behavior of the LST near the origin. For clearness of exposition, we restate that result (see [10-12]). 


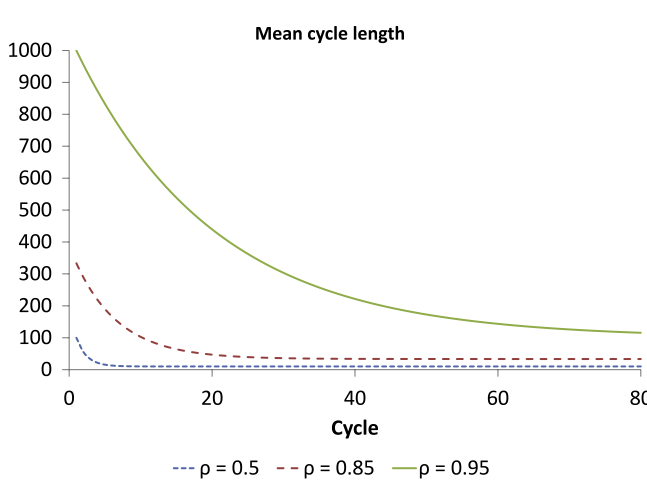

(a) Mean length per cycle.

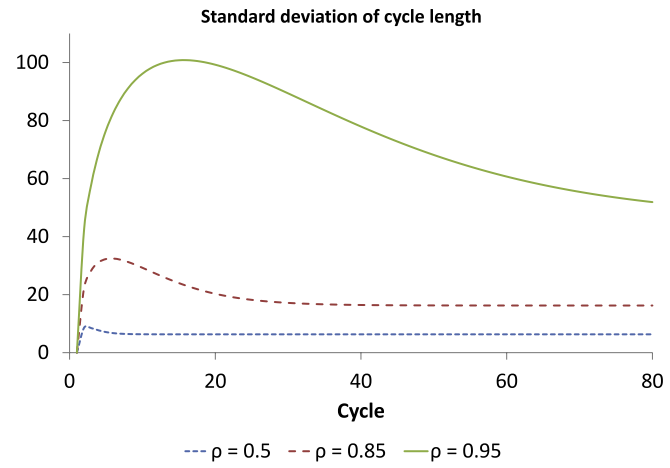

(b) Standard deviation per cycle.

Fig. 1. Mean and standard deviation per cycle, for different values of $\rho$, if the first cycle is ten times the average length.

Theorem 2. Let $X$ be a non-negative random variable, $L(\cdot)$ a slowly varying function, $v \in(m, m+1)$, and $D \geq 0$. Then the following statements are equivalent:

$$
\begin{aligned}
& \mathbb{P}(X>y)=(D+o(1)) y^{-v} L(y), \quad \text { as } y \rightarrow \infty \\
& \mathbb{E} e^{-z X}-\sum_{j=0}^{m} \mathbb{E}\left[X^{j}\right] \frac{(-z)^{j}}{j !}=(-1)^{m} \Gamma(1-v)(D+o(1)) z^{v} L(1 / z), \quad \text { as } z \downarrow 0 .
\end{aligned}
$$

To derive Proposition 3, we use the series expansion of $\mathbb{E} e^{-z C_{n}}$ and rely on Lemma 3 of [13] stating the series expansion of iterated functions that are regularly varying.

Proof of Proposition 3. We first show that $\mathbb{P}\left(C_{2}>y\right) \sim \mathbb{P}\left(C_{1}>y / \rho\right)$. The result for $C_{n}$ then follows by induction. Now, first assume that the switch-over time at the end of the second cycle can be neglected, and let the corresponding cycle length be $\tilde{C}_{n}$. Let $t(z)=\sum_{k} \delta_{k}(z)$. Then $\mathbb{E} e^{-z \tilde{C}_{2}}=\gamma_{1}(t(z))$, which is precisely an iterated function of the type considered in [13, Lemma 3]. Using the definition of $\delta_{k}(\cdot)$, we obtain $t(z)=\rho z+o(z)$. In the notation of [13], we have $\psi_{\nu}=0$ (due to Assumption A), $\phi_{v}=(-1)^{m} \Gamma(1-v)$ (due to Theorem 2), and $\psi_{1}=\rho$ (due to the series expansion of $t(z)$ above). Using [13, Lemma 3], yields

$$
\mathbb{E} e^{-z \tilde{C}_{2}}=\gamma_{1}(t(z))=\sum_{i=0}^{m} \theta_{i} z^{i}+\left((-1)^{m} \Gamma(1-v)+o(1)\right) \rho^{v} z^{v} L(1 / z) .
$$

Another application of Theorem 2 provides the desired asymptotic tail probability of $C_{2}$ in the case of zero switch-over times. From Feller [14, p. 271], and Assumption A it follows that $\mathbb{P}\left(\tilde{C}_{2}+\sum_{i} S_{i}>y\right) \sim \mathbb{P}\left(\tilde{C}_{2}>y\right)$. Hence, the switch-over times are negligible and the proof is completed.

\subsection{Numerical results}

In this section we show the impact of the first cycle on the succeeding cycles by plotting mean cycle lengths, standard deviations and correlations between different cycles. The parameters that are needed, are the first two moments of the total switch-over times, the total arrival rate and the overall load, the second moment of the service distribution of an arbitrary customer and the first two moments of the first cycle length. Let us consider the following system; switch-over times are exponentially distributed, with parameters $\mathbb{E}[S]=5$ and $\mathbb{E}\left[S^{2}\right]=50$. The total arrival rate to the system is $\Lambda=3$, the service times have an exponential distribution with $\mathbb{E}\left[B^{2}\right]=\frac{2}{3} \rho^{2}$. The first cycle is deterministic, with length $\frac{10 \mathbb{E}[S]}{1-\rho}$, so it is 10 times longer than an average cycle. The means and standard deviations of the cycle lengths for different values of $\rho$ are plotted in Fig. 1. Fig. 1(a) shows that the mean cycle length decreases to the length of an average cycle, for lower loads this decrease is faster than for higher loads. Fig. 1(b) illustrates that the standard deviation first increases and then decreases, until it converges to the standard deviation of an average cycle. The low standard deviation in the beginning is explained by the fact that the first cycle is deterministic.

The correlation between cycles 1 and $x$ is given by the covariance between cycles 1 and $x$, Eq. (11), divided by the standard deviation of cycle 1 times the standard deviation of cycle $x$. Suppose that the first cycle is an average cycle, then both standard deviations are equal, so the covariance is divided by the variance of an average cycle. It is immediately clear that the correlation between cycles 1 and $x$ is then given by $\rho^{x-1}$. This is plotted in Fig. 2. Fig. 2(a) shows that the correlation between cycles 1 and 2 is equal to $\rho$; for cycles that are further apart the correlation decreases rapidly unless the systems load is considerable. For instance, the correlation between cycles 1 and 50 is only significant if the load well exceeds 0.9 . 


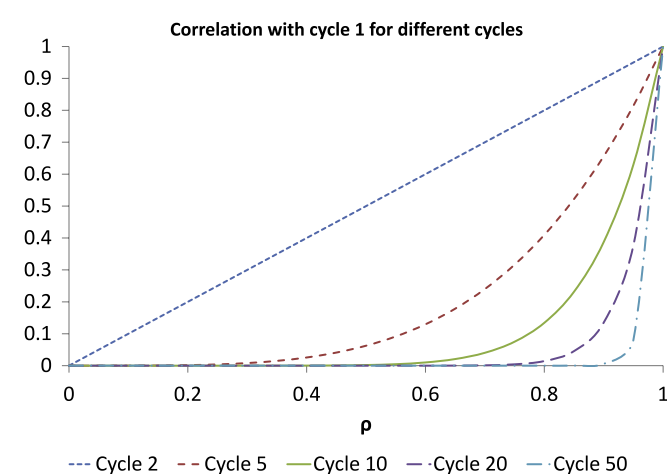

(a) Correlation as function of $\rho$.

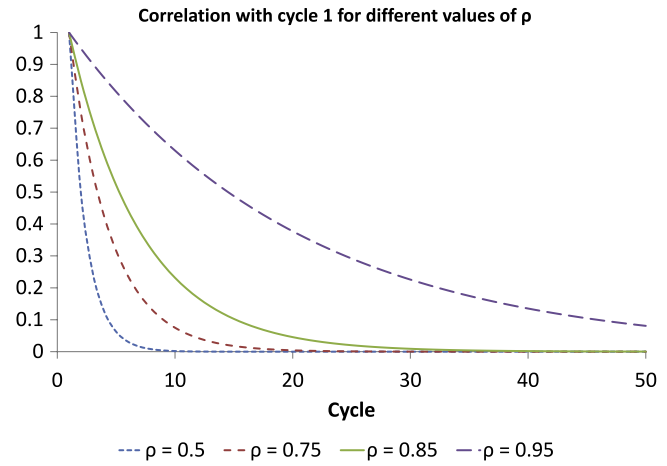

(b) Correlation as function of $x$.

Fig. 2. The correlation with cycle one, as function of $\rho$ for different values of $x$ and as function of $x$ for different values of $\rho$.

Fig. 2(b) illustrates how the correlation decreases for fixed $\rho$ by letting the distance between cycles increase. Note that when the load is equal to 1 , the correlation does not decrease, which would give a horizontal line.

\section{Gated}

First, we note that the structure and methods of proof in this section are similar to those for the globally gated case of Section 3. Recall that with the gated service policy, the server only serves the customers that were present at the queue, the moment the server arrived. Analogously to the globally gated case, we again assume that the probabilistic behavior of the first cycle is known. But for gated it is not sufficient to have information about the length of the cycle, we need information about the lengths of the first $N$ visits and switch-overs. The visit time plus the following switch-over time, i.e. $V_{i}+S_{i}$, is called the residence time. Assuming the first $N$ residence times are probabilistically known, we can write down the joint LST of the first $x$ residence times, for $x \geq N$. This LST is denoted by $\hat{\gamma}_{x}(\mathbf{z})$, and is given in the following theorem:

Theorem 3. For $x \geq N$, we have

$$
\begin{aligned}
\hat{\gamma}_{x}(\mathbf{z}) & =\mathbb{E}\left[e^{-\sum_{i=1}^{\chi} z_{i}\left(V_{i}+S_{i}\right)}\right] \\
& =\hat{\gamma}_{N}\left(z_{1}+d_{1}^{(x-1)}(\mathbf{z}), \ldots, z_{N}+d_{N}^{(x-N)}(\mathbf{z})\right) \prod_{k=1}^{x-N} S_{\tilde{k}}^{*}\left(z_{N+k}+d_{N+k}^{(x-N-k)}(\mathbf{z})\right),
\end{aligned}
$$

with $\tilde{x}=x \bmod N$ and

$$
d_{n}^{(i)}(\mathbf{z})=\sum_{k=n+1}^{N+n} \delta_{\tilde{k}}\left(z_{k}+d_{k}^{(i+n-k)}(\mathbf{z})\right) \mathbb{1}_{\{N<k \leq n+i\}} .
$$

Note that the vector $\mathbf{z}$ has length $x$, and every element $z_{i}$ of the vector now corresponds to the $i$ th residence time for $i=1, \ldots, x$. The recursive term $d_{n}^{(i)}(\mathbf{z})$ is similar to the $\zeta$ of the globally gated case. In order to prove Theorem 3 , we first establish the following lemma (with the proof deferred to Appendix A).

Lemma 2. For $n=1,2, \ldots$ fixed and $x \geq n$ and $x \geq N$, we have

$$
\left(z_{n}^{\prime}+d_{n}^{(x-n)}\left(\mathbf{z}^{\prime}\right)\right)=z_{n}+d_{n}^{(x-n+1)}(\mathbf{z}),
$$

with $\mathbf{z}^{\prime}=\mathbf{z}+d_{x}^{(1)}(\mathbf{z}) \sum_{i=x-N+1}^{x} \mathbf{e}_{i}$.

Proof of Theorem 3. For the proof of Theorem 3, we use induction. First we take the case $x=N$, giving

$$
\begin{aligned}
\hat{\gamma}_{N}(\mathbf{z}) & =\hat{\gamma}_{N}\left(z_{1}+d_{1}^{(N-1)}(\mathbf{z}), \ldots, z_{N}+d_{N}^{(N-N)}(\mathbf{z})\right) \prod_{k=1}^{0} S_{\tilde{k}}^{*}\left(z_{N+k}+d_{N+k}^{(-k)}(\mathbf{z})\right) \\
& =\hat{\gamma}_{N}\left(z_{1}, \ldots, z_{N}\right),
\end{aligned}
$$


because $d_{n}^{(N-n)}(\mathbf{z})=0$ for all $n$, and the product of switch-overs is empty and thus equals 1 . Next we assume that Theorem 3 holds for all $k \leq x$, then for $k=x+1$, we have

$$
\begin{aligned}
\hat{\gamma}_{x+1}(\mathbf{z}) & =\hat{\gamma}_{x}\left(z_{1}, \ldots, z_{x-N}, z_{x-N+1}+d_{x}^{(1)}, \ldots, z_{x}+d_{x}^{(1)}\right) S_{x+1}^{*}\left(z_{x+1}\right) \\
& =\left(\hat{\gamma}_{N}\left(z_{1}^{\prime}+d_{1}^{(x-1)}\left(\mathbf{z}^{\prime}\right), \ldots, z_{N}^{\prime}+d_{N}^{(x-N)}\left(\mathbf{z}^{\prime}\right)\right) \prod_{k=1}^{x-N} S_{\widetilde{k}}^{*}\left(z_{N+k}^{\prime}+d_{N+k}^{(x-N-k)}\left(\mathbf{z}^{\prime}\right)\right)\right) S_{x+1}^{*}\left(z_{x+1}\right) \\
& =\hat{\gamma}_{N}\left(z_{1}+d_{1}^{(x)}(\mathbf{z}), \ldots, z_{N}+d_{N}^{(x-N+1)}(\mathbf{z})\right) \prod_{k=1}^{x-N} S_{\tilde{k}}^{*}\left(z_{N+k}+d_{N+k}^{(x-N-k+1)}(\mathbf{z})\right) S_{x+1}^{*}\left(z_{x+1}\right) \\
& =\hat{\gamma}_{N}\left(z_{1}+d_{1}^{(x)}(\mathbf{z}), \ldots, z_{N}+d_{N}^{(x-N+1)}(\mathbf{z})\right) \prod_{k=1}^{x-N+1} S_{\tilde{k}}^{*}\left(z_{N+k}+d_{N+k}^{(x-N-k+1)}(\mathbf{z})\right) .
\end{aligned}
$$

For the first equality, we use the same reasoning that was used for Eq. (2). For the second equality, the induction hypothesis is used. The third equality uses Lemma 2. For the last equality, note that $\overline{x+1}=x+1 \bmod N$, which equals $\overline{x-N+1}=$ $x-N+1 \bmod N$. This completes the proof.

As in Section 3, we define $J \subseteq\{0,1,2, \ldots, x\}$ as the set of residence times we wish to include. Specifically, we take $z_{j}$ equal to $z$ if $j \in J$, and 0 otherwise. For example, if we want to calculate the distribution of the length of the first residence time in the second cycle we set $z_{N+1}=z$ and all other $z_{i}$ equal to 0 . Moments can now again be derived by differentiating with respect to $z$. The derivative of $\hat{\gamma}_{x}$ with respect to $z$ (with $z_{i}$ either $z$ or 0 ) is then given by:

$$
\begin{aligned}
\frac{d}{d z} \hat{\gamma}_{x}(\mathbf{z})= & \sum_{j=1}^{N} \hat{\gamma}_{N}^{(j)}\left(z_{1}+d_{1}^{(x-1)}(\mathbf{z}), \ldots, z_{N}+d_{1}^{(x-N)}(\mathbf{z})\right)\left(\mathbb{1}_{\{j \in J\}}+\frac{d}{d z} d_{j}^{(x-j)}(\mathbf{z})\right) \prod_{k=1}^{x-N} S_{\tilde{k}}^{*}\left(z_{N+k}+d_{N+k}^{(x-N-k)}(\mathbf{z})\right) \\
& +\hat{\gamma}_{N}\left(z_{1}+d_{1}^{(x-1)}(\mathbf{z}), \ldots, z_{N}+d_{N}^{(x-N)}(\mathbf{z})\right) \sum_{j=1}^{x-N} \frac{d}{d z} S_{\tilde{j}}^{*}\left(z_{N+j}+d_{N+j}^{(x-N-j)}(\mathbf{z})\right) \\
& \times\left(\mathbb{1}_{\{N+j \in J\}}+\frac{d}{d z} d_{N+j}^{(x-N-j)}(\mathbf{z})\right) \prod_{k \neq j} S_{\tilde{k}}^{*}\left(z_{N+k}+d_{N+k}^{(x-N-k)}(\mathbf{z})\right),
\end{aligned}
$$

where $\hat{\gamma}_{N}^{(j)}(\cdot)$ is the partial derivative of $\hat{\gamma}_{N}(\cdot)$, with respect to the $j$ th parameter. The derivative of $d_{j}^{(x-j)}(\mathbf{z})$ is recursively defined as

$$
\frac{d}{d z} d_{j}^{(x-j)}(\mathbf{z})=\sum_{k=j+1}^{N+j} \frac{d}{d z} \delta_{\tilde{k}}\left(z_{k}+d_{k}^{(x-k)}(\mathbf{z})\right)\left(\mathbb{1}_{\{k \in J\}}+\frac{d}{d z} d_{k}^{(x-k)}(\mathbf{z})\right) \mathbb{1}_{\{N<k \leq x\}} .
$$

For convenience, we define $\alpha_{j}=\left.\frac{d}{d z} d_{j}^{(x-j)}(\mathbf{z})\right|_{\{z=0\}}$. The expected length of residence times under consideration (that is, in set $J$ ) is given by the following proposition.

Proposition 4 (First Moments). The expected total residence time for set $J$ is

$$
\mathbb{E}\left[\sum_{j \in J} V_{j}+S_{j}\right]=\sum_{j=1}^{N}\left(\mathbb{E}\left[\hat{V}_{j}\right]+\mathbb{E}\left[\hat{S}_{j}\right]\right)\left(\mathbb{1}_{\{j \in J\}}+\alpha_{j}\right)+\sum_{j=1}^{x-N} \mathbb{E}\left[S_{j}\right]\left(\mathbb{1}_{\{N+j \in J\}}+\alpha_{N+j}\right),
$$

with

$$
\alpha_{j}=\sum_{k=j+1}^{N+j} \rho_{\tilde{k}}\left(\mathbb{1}_{\{k \in J\}}+\alpha_{k}\right) \mathbb{1}_{\{N<k \leq x\}} .
$$

Example 1. An interesting special case is where we are only interested in two subsequent cycles, i.e. $x=2 N$. Take $J=\{N+1, \ldots, x\}$. From (15) it may be easily verified that

$$
\alpha_{i}=\sum_{j=N+1}^{N+i} \rho_{\tilde{j}}+\rho_{\tilde{j}} \sum_{k=j+1}^{x} \rho_{\tilde{k}} \prod_{l=j+1}^{k-1}\left(1+\rho_{\tilde{l}}\right) .
$$

For the second moment we need to differentiate Eq. (13), the result can be found in Appendix B, Eq. (21). Taking $z=0$ yields the second moments. 
Proposition 5 (Second Moments). The second moment of the total residence time for set $J$ is

$$
\begin{aligned}
\mathbb{E}\left[\left(\sum_{j \in J}\left(V_{j}+S_{j}\right)\right)^{2}\right]= & \sum_{j=1}^{N} \sum_{l=1}^{N} \mathbb{E}\left[\left(\hat{V}_{j}+\hat{S}_{j}\right)\left(\hat{V}_{l}+\hat{S}_{l}\right)\right]\left(\mathbb{1}_{\{l \in J\}}+\alpha_{l}\right)\left(\mathbb{1}_{\{j \in J\}}+\alpha_{j}\right) \\
& -\left.\sum_{j=1}^{N} \mathbb{E}\left[\left(\hat{V}_{j}+\hat{S}_{j}\right)\right] \frac{d^{2}}{d z^{2}} d_{j}^{(x-j)}(\mathbf{z})\right|_{\{z=0\}} \\
& +2 \sum_{j=1}^{N} \mathbb{E}\left[\left(\hat{V}_{j}+\hat{S}_{j}\right)\right]\left(\mathbb{1}_{\{j \in J\}}+\alpha_{j}\right) \sum_{k=1}^{x-N} \mathbb{E}\left[S_{\tilde{k}}\right]\left(\mathbb{1}_{\{N+k \in J\}}+\alpha_{N+k}\right) \\
& +\sum_{j=1}^{x-N}\left[\mathbb{E}\left[S_{\tilde{j}}^{2}\right]\left(\mathbb{1}_{\{N+j \in J\}}+\alpha_{N+j}\right)^{2}-\left.\mathbb{E}\left[S_{\tilde{j}}\right] \frac{d^{2}}{d z^{2}} d_{N+j}^{(x-N-j)}(\mathbf{z})\right|_{\{z=0\}}\right. \\
& \left.+\mathbb{E}\left[S_{\tilde{j}}\right]\left(\mathbb{1}_{\{N+j \in J\}}+\alpha_{N+j}\right) \sum_{k \neq j} \mathbb{E}\left[S_{\tilde{k}}\right]\left(\mathbb{1}_{\{N+k \in J\}}+\alpha_{N+k}\right)\right],
\end{aligned}
$$

with $\alpha_{j}$ given by (15) and $\left.\frac{d^{2}}{d z^{2}} d_{j}^{(x-j)}(\mathbf{z})\right|_{\{z=0\}}$ recursively given by

$$
\left.\frac{d^{2}}{d z^{2}} d_{j}^{(x-j)}(\mathbf{z})\right|_{\{z=0\}}=\sum_{k=j+1}^{N+j}\left[-\lambda_{\tilde{k}} \mathbb{E}\left[B_{\tilde{k}}^{2}\right]\left(\mathbb{1}_{\{k \in J\}}+\alpha_{k}\right)^{2}+\left.\rho_{\tilde{k}} \frac{d^{2}}{d z^{2}} d_{k}^{(x-k)}(\mathbf{z})\right|_{\{z=0\}}\right] \mathbb{1}_{\{N<k \leq x\}}
$$

Remark 3 (Comparison with Globally Gated). For globally gated we are able to find closed-form expressions for both the mean and the second moment. For gated this turned out to be involved, so the recursive terms are left intact. Assuming the initial cycle to be in steady-state, the relations between the residence times of the second cycle expressed in terms of the first cycle give rise to a system of equations. We refer to e.g. Takagi [15] for such an approach for the analysis of the number of customers at polling instants.

By adding more parameters to the joint LST of the residence times, the visit times and switch-over times can be tracked separately. Analogously to the gated case, the globally gated case can be extended to also record the visit and switch-over times per cycle. This can be useful, for example, for determining the output process.

\subsection{Asymptotic properties}

The asymptotic results are similar to those for the globally gated case. However, the expressions are more involved and concern iteratively defined functions. Below, we focus on the two most interesting cases: heavy traffic and heavy-tailed initial cycle length.

Heavy traffic. For the heavy-traffic regime, we consider the usual scaling of residence times $\tilde{R}_{i}:=\tilde{V}_{i}+\tilde{S}_{i}=(1-\rho)\left(V_{i}+S_{i}\right)$. For each variable $x$ that is a function of $\rho$, we denote its value evaluated at $\rho=1$ by $\hat{x}$.

Proposition 6. For the joint LST of scaled residence times, we have

$$
\lim _{\rho \uparrow 1} \hat{\gamma}_{x}(\mathbf{z}(1-\rho))=\hat{\gamma}_{N}^{\mathrm{HT}}\left(z_{1}+\hat{\alpha}_{1}, \ldots, z_{N}+\hat{\alpha}_{N}\right),
$$

with $\hat{\gamma}_{N}^{\mathrm{HT}}(\cdot)$ the joint transform of scaled initial residence times, and $\hat{\alpha}_{j}$ given by the recursion

$$
\hat{\alpha}_{j}=\sum_{k=j+1}^{N+j} \hat{\rho}_{\tilde{k}}\left(z_{k}+\hat{\alpha}_{k}\right) \mathbb{1}_{\{N<k \leq x\}} .
$$

Proof. The heavy-traffic limit follows from

$$
\begin{aligned}
\lim _{\rho \uparrow 1} \hat{\gamma}_{x}(\mathbf{z}(1-\rho))= & \lim _{\rho \uparrow 1} \hat{\gamma}_{N}\left(z_{1}(1-\rho)+d_{1}^{(x-1)}(\mathbf{z}(1-\rho)), \ldots, z_{N}(1-\rho)+d_{N}^{(x-N)}(\mathbf{z}(1-\rho))\right) \\
& \times \prod_{k=1}^{x-N} S_{\tilde{k}}^{*}\left(z_{N+k}(1-\rho)+d_{N+k}^{(x-N-k)}(\mathbf{z}(1-\rho))\right) .
\end{aligned}
$$


Using Theorem 3 and by applying l'Hôpital's rule, we obtain

$$
\lim _{\rho \uparrow 1} \frac{d_{n}^{(i)}(\mathbf{z}(1-\rho))}{1-\rho}=\lim _{\rho \uparrow 1} \sum_{k=n+1}^{N} \delta_{\tilde{k}}^{\prime}\left(z_{k}(1-\rho)+d_{k}^{(i+n-k)}(\mathbf{z}(1-\rho))\right)\left(z_{k}+\frac{\mathrm{d}}{\mathrm{d}(1-\rho)} d_{k}^{(i+n-k)}(\mathbf{z}(1-\rho))\right) \mathbb{1}_{\{N<k \leq n+i\}} .
$$

The recursion (17) then follows by letting $\hat{\alpha}_{j}=\lim _{\rho \uparrow 1} d_{n}^{(i)}(\mathbf{z}(1-\rho)) /(1-\rho)$. Combining the above provides the result.

Proposition 6 shows that in heavy traffic, the joint LST of residence times is fully characterized by the LST of the initial cycle. As such, we see that an 'averaging principle' applies to our transient results, as the future evolution of residence times are specified by average input values. However, we note that, depending on the composition of the first cycle, the scaled cycle lengths may either increase or decrease; see Example 2 for an illustration.

Example 2. For simplicity, take $N=2$ and $x=4$. Similar to (16), we may obtain that $\hat{\alpha}_{1}=\hat{\rho}_{1} z_{3}+\hat{\rho}_{1} \hat{\rho}_{2} z_{4}$ and $\hat{\alpha}_{2}=\hat{\rho}_{1} z_{3}+\left(1+\hat{\rho}_{1}\right) \hat{\rho}_{2} z_{4}$, yielding

$$
\lim _{\rho \uparrow 1} \hat{\gamma}_{x}(\mathbf{z}(1-\rho))=\mathbb{E}\left[\exp \left\{-z_{1} \tilde{R}_{1}-z_{2} \tilde{R}_{2}-z_{3} \hat{\rho}_{1}\left(\tilde{R}_{1}+\tilde{R}_{2}\right)-z_{4} \hat{\rho}_{2}\left(\hat{\rho}_{1} \tilde{R}_{1}+\left(1+\hat{\rho}_{1}\right) \tilde{R}_{2}\right)\right\}\right] .
$$

Addressing expected values, it holds in equilibrium that $\left(1-\hat{\rho}_{1}\right) \mathbb{E}\left[\tilde{R}_{1}^{*}\right]=\hat{\rho}_{1} \mathbb{E}\left[\tilde{R}_{2}^{*}\right]$. If we assume that the initial residence time of queue 1 is relatively short, i.e., that $\left(1-\hat{\rho}_{1}\right) \mathbb{E}\left[\tilde{R}_{1}^{*}\right] \leq \hat{\rho}_{1} \mathbb{E}\left[\tilde{R}_{2}^{*}\right]$, then it may be verified that $\mathbb{E}\left[\tilde{R}_{3}\right] \geq \mathbb{E}\left[\tilde{R}_{1}\right]$ and $\mathbb{E}\left[\tilde{R}_{4}\right] \leq \mathbb{E}\left[\tilde{R}_{2}\right]$. Moreover, for the total cycle length we have $\mathbb{E}\left[\tilde{R}_{3}+\tilde{R}_{4}\right] \geq \mathbb{E}\left[\tilde{R}_{1}+\tilde{R}_{2}\right]$. This means that the residence times converge to their equilibrium value, whereas the overall cycle length is increasing. For a relatively long initial residence time of queue 1, i.e., $\left(1-\hat{\rho}_{1}\right) \mathbb{E}\left[\tilde{R}_{1}^{*}\right] \geq \hat{\rho}_{1} \mathbb{E}\left[\tilde{R}_{2}^{*}\right]$, all inequalities are reversed.

Heavy-tailed initial cycle length. Clearly, an excessively long residence time affects subsequent residence times. For convenience, we assume here that queue $N$ (which may be arbitrary) has a heavy-tailed residence time. When the residence time of queue $i<N$ would have a heavy tail, this would affect the tail behavior of queues $i+1, \ldots, N$ as well, which is precisely the effect we aim to study. More precisely, we assume that the residence time of queue $N$ is regular varying of index $-v$ (with $m<v<m+1$ ) and asymptotically dominates the tail of the residence times at queues $1, \ldots, N-1$, that is

$$
\mathbb{P}\left(V_{1}+S_{1}>\eta_{1} y, \ldots, V_{N-1}+S_{N-1}>\eta_{N-1} y, V_{N}+S_{N}>y\right) \sim \mathbb{P}\left(V_{N}+S_{N}>y\right) .
$$

This leads to the following assumption:

Assumption B. The initial cycle length is asymptotically dominated by the residence time of queue $N$, giving

$$
\hat{\gamma}_{N}\left(f_{1}(z), \ldots, f_{N-1}(z), z\right)=\sum_{i=0}^{m} a_{i} z^{i}+(-1)^{m} \Gamma(1-v)(1+o(1)) z^{v} L(1 / z) .
$$

As in the globally gated case, it holds that an excessively long $n$th residence time is most likely due to an excessively long initial visit of queue $N$, while the system shows average behavior otherwise. The average behavior is specified by a similar recursive scheme as for the mean residence times and heavy-traffic asymptotics.

Proposition 7. Suppose that $V_{N} \in \mathcal{R}_{-\nu}$ and Assumptions A and B are satisfied. Then,

$$
\mathbb{P}\left(V_{x}+S_{x}>y\right) \sim \mathbb{P}\left(V_{N}>y / \tilde{\alpha}_{N}\right),
$$

with $\tilde{\alpha}_{x}=1$ and $\tilde{\alpha}_{N}$ given by the recursion

$$
\tilde{\alpha}_{j}=\sum_{k=j+1}^{N+j} \rho_{\tilde{k}} \tilde{\alpha}_{k} \mathbb{1}_{\{N<k \leq x\}} .
$$

Proof. The proof is along the same line as the proof of Proposition 3. Consider the visit of queue $x$, i.e., take $z_{x}=z$ and $z_{i}=0$ for $i=1, \ldots, x-1$. First, we assume that all switch-over times equal zero, represented by $\tilde{V}_{j}$ in the notation for the visit time of the $j$ th queue. From Theorem 3 , we obtain $\mathbb{E} e^{-z \tilde{V}_{x}}=\hat{\gamma}_{N}\left(d_{1}^{(x-1)}, \ldots, d_{N}^{(x-N)}\right)$. We thus need to consider the series expansion of the terms $d_{j}^{(x-j)}$, which are in fact iterated functions. In view of [13, Lemma 3] and Assumption A, we have $\psi_{v}=0$ (in the notation of [13]; see also the proof of Proposition 3) and we only need to consider the first term of the series expansion of $d_{j}^{(x-j)}$. Using Theorem 3, it easily follows that $d_{j}^{(x-j)}=\tilde{\alpha}_{j} z+o(z)$ where $\tilde{\alpha}_{j}$ follows from the recursion (18). Applying the same arguments as in [13, Lemma 3] combined with Assumption B, we obtain

$$
\mathbb{E} e^{-z \tilde{V}_{x}}=\hat{\gamma}_{N}\left(d_{1}^{(x-1)}, \ldots, d_{N}^{(x-N)}\right)=\sum_{i=0}^{m} \theta_{i} z^{i}+\left((-1)^{m} \Gamma(1-v)+o(1)\right) \tilde{\alpha}_{N}^{v} z^{v} L(1 / z) .
$$




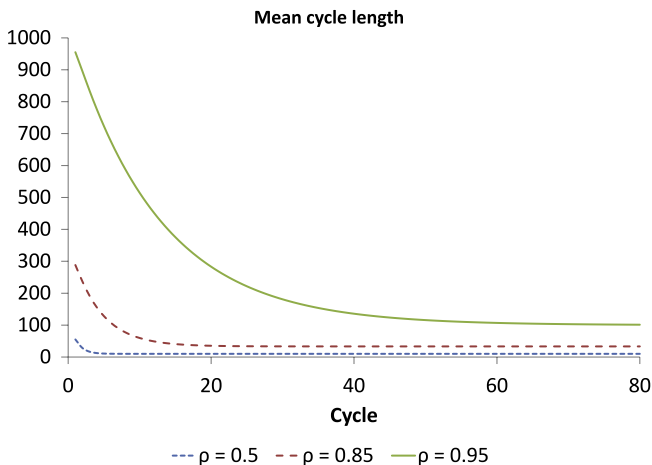

(a) Mean length per cycle.

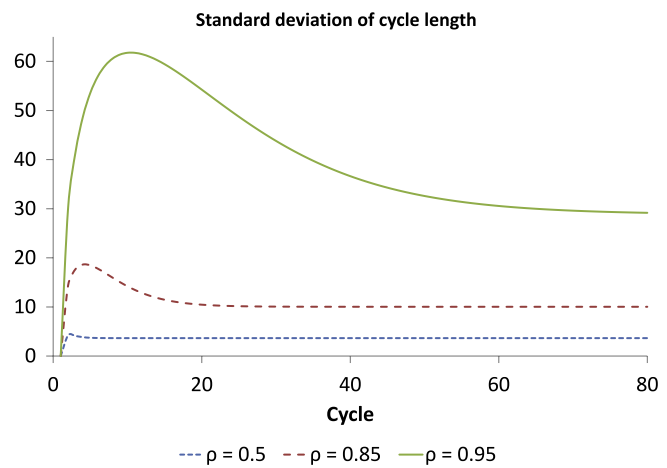

(b) Standard deviation per cycle.

Fig. 3. Mean and standard deviation per cycle, for different values of $\rho$, if the first cycle is ten times normal length and deterministic.

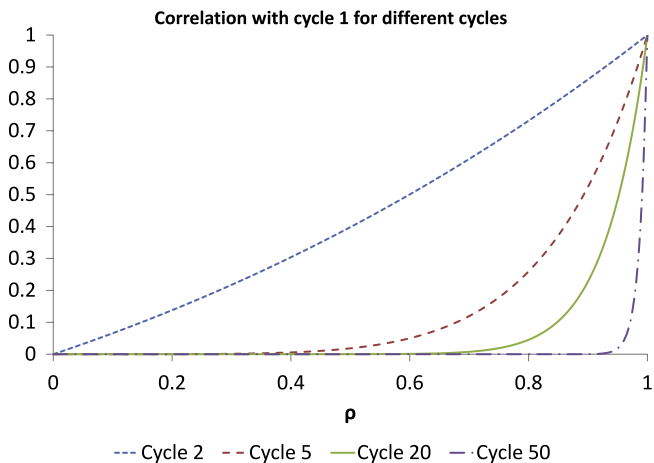

(a) Correlation as function of $\rho$.

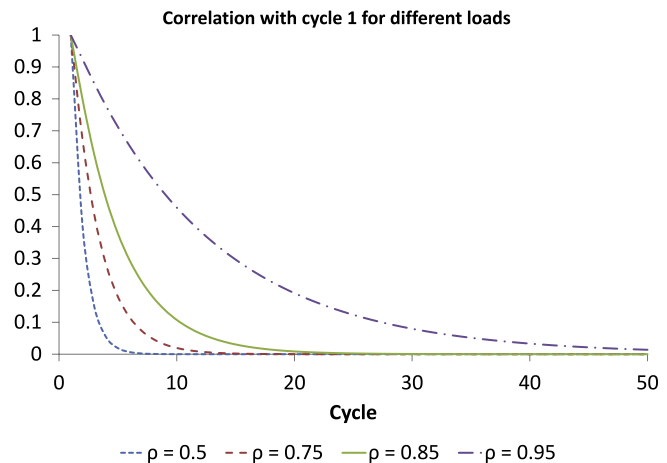

(b) Correlation as function of $x$.

Fig. 4. The correlation with cycle one, as function of $\rho$ for different values of $x$ and as function of $x$ for different values of $\rho$.

Using Theorem 2 yields $\mathbb{P}\left(\tilde{V}_{x}>y\right) \sim \mathbb{P}\left(V_{N}>y / \tilde{\alpha}_{N}\right)$. Again, under Assumption $\mathrm{A}$, it is straightforward to show that the contribution of switch-over times and the contribution of work arriving during these switch-over times are negligible. This completes the proof.

\subsection{Numerical results}

In this section we use a numerical example to illustrate the properties of a gated system. Consider a symmetric system with $N=4$ queues, $\lambda_{i}=1, \mathbb{E}\left[B_{i}\right]=\rho_{i}, \mathbb{E}\left[B_{i}^{2}\right]=2 \rho_{i}^{2}, \rho_{i}=\rho / N, \mathbb{E}\left[S_{i}\right]=\frac{5}{4}$, and $\mathbb{E}\left[S_{i}^{2}\right]=\frac{25}{8}$, for all $i$. Because of a disaster, all visit times take ten times longer than average, but switch-over times are not affected, so $\mathbb{E}\left[\left(\hat{V}_{i}+\hat{S}_{i}\right)\right]=10 \frac{\rho \mathbb{E}\left[S_{i}\right]}{1-\rho}+\mathbb{E}\left[S_{i}\right]$. We take the residence times deterministic, giving $\mathbb{E}\left[\left(\hat{V}_{i}+\hat{S}_{i}\right)\left(\hat{V}_{j}+\hat{S}_{j}\right)\right]=\mathbb{E}\left[\left(\hat{V}_{i}+\hat{S}_{i}\right)\right]^{2}$, for $i, j=1,2,3,4$.

The mean and standard deviation of the cycle lengths of this system are plotted in Fig. 3. We see that the figures look very similar to the globally gated case in Fig. 1. Fig. 4 shows the correlation between cycle 1 and various other cycles, where the first cycle is distributed as an average cycle. These figures also look similar to the globally gated case in Fig. 2. Recall that the correlation between cycle 1 and 2 was equal to $\rho$ in the globally gated system, Fig. 4(a) shows that this is not the case for the gated system, however, the difference is small.

\section{Acknowledgment}

We would like to thank Erik Winands for useful suggestions and fruitful discussions.

\section{Appendix A. Proofs}

Proof of Lemma 1. We prove Lemma 1 by induction. First we show that the lemma holds for $i=2$ :

$$
\zeta_{n}^{(2)}\left(\mathbf{z}^{\prime}\right)=\sum_{k=1}^{N} \delta_{k}\left(z_{n+1}+\zeta_{n+1}^{(2)}(\mathbf{z})+\zeta_{n+1}^{(1)}(\mathbf{z})\right)=\zeta_{n}^{(3)}(\mathbf{z}) .
$$


Both equalities follow from the definition of $\zeta$, given in Eq. (4). Now assume that (5) is true for all $k \leq i$. We have for $k=i+1$ :

$$
\begin{aligned}
\zeta_{n}^{(i+1)}\left(\mathbf{z}^{\prime}\right) & =\sum_{k=1}^{N} \delta_{k}\left(z_{n+1}+\zeta_{n+1}^{(i)}\left(\mathbf{z}^{\prime}\right)\right) \\
& =\sum_{k=1}^{N} \delta_{k}\left(z_{n+1}+\zeta_{n+1}^{(i+1)}(\mathbf{z})\right)=\zeta_{n}^{(i+2)}(\mathbf{z}) .
\end{aligned}
$$

The first and the last equality use the definition of $\zeta$, and for the second equality, the induction hypotheses is used. This completes the proof.

Proof of Lemma 2. We prove Lemma 2 using induction. First we show that the lemma holds for $n=x$, because then $x-n=0$, providing

$$
\left(z_{x}^{\prime}+d_{x}^{(0)}\left(\mathbf{z}^{\prime}\right)\right)=z_{x}+d_{x}^{(1)}(\mathbf{z})+0 .
$$

This follows from the fact that $z_{x}$ is the last element of $\mathbf{z}$ and $d_{x}^{(0)}=0$. Now assume that Eq. (12) holds for all $k \leq x-n$. For $k+1=x-n$, we need to consider two cases: (i) the case where $z_{x-k-1}$ is not one of the last $N$ elements of $\mathbf{z}$ and (ii) the case where it is. For case (i) we have $x-k-1<x-N+1$, thus $k>N-2$, giving

$$
\begin{aligned}
\left(z_{x-k-1}^{\prime}+d_{x-k-1}^{(k+1)}\left(\mathbf{z}^{\prime}\right)\right) & =z_{x-k-1}+\sum_{l=x-k}^{N+x-k-1} \delta_{l}\left(z_{l}^{\prime}+d_{l}^{(x-l)}\left(\mathbf{z}^{\prime}\right)\right) \\
& =z_{x-k-1}+\sum_{l=x-k}^{N+x-k-1} \delta_{I}\left(z_{l}+d_{l}^{(x-l+1)}(\mathbf{z})\right) \mathbb{1}_{\{N<l \leq x\}} \\
& =z_{x-k-1}+d_{x-k-1}^{(k+2)}(\mathbf{z}) .
\end{aligned}
$$

For the first equality, the definition of $d_{n}^{(i)}(\mathbf{z})$ is used, the second equality uses the induction hypotheses and the final equality uses the definition again. Note that $N+x-k-1 \leq x$ and thus also $N+x-k-1 \leq x+1$, so the last equality is indeed true. For the second case, we have

$$
\begin{aligned}
\left(z_{x-k-1}^{\prime}+d_{x-k-1}^{(k+1)}\left(\mathbf{z}^{\prime}\right)\right) & =z_{x-k-1}+d_{x}^{(1)}(\mathbf{z})+\sum_{l=x-k}^{N+x-k-1} \delta_{\tilde{l}}\left(z_{l}^{\prime}+d_{l}^{(x-l)}\left(\mathbf{z}^{\prime}\right)\right) \mathbb{1}_{\{N<l \leq x\}} \\
& =z_{x-k-1}+\delta_{\widetilde{x+1}}\left(z_{x+1}+d_{x+1}^{(0)}(\mathbf{z})\right)+\sum_{l=x-k}^{N+x-k-1} \delta_{\tilde{l}}\left(z_{l}+d_{l}^{(x-l+1)}(\mathbf{z})\right) \mathbb{1}_{\{N<l \leq x\}} \\
& =z_{x-k-1}+\sum_{l=x-k}^{N+x-k-1} \delta_{\tilde{l}}\left(z_{l}+d_{l}^{(x-l+1)}(\mathbf{z})\right) \mathbb{1}_{\{N<l \leq x+1\}}=z_{x-k-1}+d_{x-k-1}^{(k+2)}(\mathbf{z}) .
\end{aligned}
$$

The first equality uses the definition of $d_{n}^{(i)}(\mathbf{z})$, whereas the second equality also uses the induction hypothesis. For the third equality we use the fact that the indicator function determines the end of the summation at $x$ and the extra term contains the case were $k=x+1$. The final equality follows again from the definition, completing the proof.

\section{Appendix B. Second derivatives}

For globally gated, the second derivative of $\gamma_{x}(\mathbf{z})$ given in Eq. (3), is given by

$$
\begin{aligned}
\frac{d^{2}}{d z^{2}} \gamma_{x}(\mathbf{z})= & \frac{d^{2}}{d z^{2}} \gamma_{1}\left(z_{1}+\zeta_{1}^{(x)}(\mathbf{z})\right)\left(\mathbb{1}_{\{1 \in J\}}+\frac{d}{d z} \zeta_{1}^{(x)}(\mathbf{z})\right) \prod_{j=1}^{2} S^{*}\left(z_{j+1}+\zeta_{j+1}^{(x-j)}(\mathbf{z})\right)+2 \frac{d}{d z} \gamma_{1}\left(z_{1}+\zeta_{1}^{(x)}(\mathbf{z})\right) \\
& \times\left(\mathbb{1}_{\{1 \in J\}}+\frac{d}{d z} \zeta_{1}^{(x)}(\mathbf{z})\right) \sum_{j=1}^{x-1} \frac{d}{d z} S^{*}\left(z_{j+1}+\zeta_{j+1}^{(x-j)}(\mathbf{z})\right)\left(\mathbb{1}_{\{j+1 \in J\}}+\frac{d}{d z} \zeta_{j+1}^{(x-j)}(\mathbf{z})\right) \\
& \times \prod_{k \neq j}^{x-1} S^{*}\left(z_{k+1}+\zeta_{k+1}^{(x-k)}(\mathbf{z})\right)+\frac{d}{d z} \gamma_{1}\left(z_{1}+\zeta_{1}^{(x)}(\mathbf{z})\right) \frac{d^{2}}{d z^{2}} \zeta_{1}^{(x)}(\mathbf{z}) \\
& \times \prod_{j=1}^{x-1} S^{*}\left(z_{j+1}+\zeta_{j+1}^{(x-j)}(\mathbf{z})\right)+\gamma_{1}\left(z_{1}+\zeta_{1}^{(x)}(\mathbf{z})\right) \sum_{j=1}^{x-1}\left[\frac{d^{2}}{d z^{2}} S^{*}\left(z_{j+1}+\zeta_{j+1}^{(x-j)}(\mathbf{z})\right)\right.
\end{aligned}
$$




$$
\begin{aligned}
& \times\left(\mathbb{1}_{\{j+1 \in J\}}+\frac{d}{d z} \zeta_{j+1}^{(x-j)}(\mathbf{z})\right) \prod_{k \neq j}^{2 x-1} S^{*}\left(z_{k+1}+\zeta_{k+1}^{(x-k)}(\mathbf{z})\right)+\frac{d}{d z} S^{*}\left(z_{j+1}+\zeta_{j+1}^{(x-j)}(\mathbf{z})\right) \frac{d^{2}}{d z^{2}} \zeta_{j+1}^{(x-j)}(\mathbf{z}) \\
& \times \prod_{k \neq j}^{x-1} S^{*}\left(z_{k+1}+\zeta_{k+1}^{(x-k)}(\mathbf{z})\right)+\frac{d}{d z} S^{*}\left(z_{j+1}+\zeta_{j+1}^{(x-j)}(\mathbf{z})\right)\left(\mathbb{1}_{\{j+1 \in J\}}+\frac{d}{d z} \zeta_{j+1}^{(x-j)}(\mathbf{z})\right) \\
& \left.\times \sum_{k \neq j}^{x-1} \frac{d}{d z} S^{*}\left(z_{k+1}+\zeta_{k+1}^{(x-k)}(\mathbf{z})\right)\left(\mathbb{1}_{\{k+1 \in J\}}+\frac{d}{d z} \zeta_{k+1}^{(x-k)}(\mathbf{z})\right) \prod_{l \neq k}^{x-1} S^{*}\left(z_{l+1}+\zeta_{l+1}^{(x-l)}(\mathbf{z})\right)\right] .
\end{aligned}
$$

The second derivative of $\zeta_{n}^{(i)}(\mathbf{z})$, given in (4), is equal to

$$
\frac{d^{2}}{d z^{2}} \zeta_{n}^{(i)}(\mathbf{z})=\sum_{k=1}^{N}\left[\frac{d^{2}}{d z^{2}} \delta_{k}\left(z_{n+1}+\zeta_{n+1}^{(i-1)}(\mathbf{z})\right)\left(\mathbb{1}_{\{n+1 \in J\}}+\frac{d}{d z} \zeta_{n+1}^{(i-1)}(\mathbf{z})\right)^{2}+\frac{d}{d z} \delta_{k}\left(z_{n+1}+\zeta_{n+1}^{(i-1)}(\mathbf{z})\right) \frac{d^{2}}{d z^{2}} \zeta_{n+1}^{(i-1)}(\mathbf{z})\right] .
$$

For gated, the second derivative of $\hat{\gamma}_{x}(\mathbf{z})$, given in Theorem 3 , is given by

$$
\begin{aligned}
\frac{d^{2}}{d z^{2}} \hat{\gamma}_{x}(\mathbf{z})= & \sum_{j=1}^{N} \sum_{l=1}^{N} \hat{\gamma}_{N}^{(j, l)}\left(z_{1}+d_{1}^{(x-1)}(\mathbf{z}), \ldots, z_{N}+d_{1}^{(x-N)}(\mathbf{z})\right)\left(\mathbb{1}_{\{l \in J\}}+\frac{d}{d z} d_{l}^{(x-l)}(\mathbf{z})\right) \\
& \times\left(\mathbb{1}_{j \in J\}}+\frac{d}{d z} d_{j}^{(x-j)}(\mathbf{z})\right) \prod_{k=1}^{x-N} S_{\tilde{k}}^{*}\left(z_{N+k}+d_{N+k}^{(x-N-k)}(\mathbf{z})\right) \\
& +\sum_{j=1}^{N} \hat{\gamma}_{N}^{(j)}\left(z_{1}+d_{1}^{(x-1)}(\mathbf{z}), \ldots, z_{N}+d_{1}^{(x-N)}(\mathbf{z})\right) \frac{d^{2}}{d z^{2}} d_{j}^{(x-j)}(\mathbf{z}) \prod_{k=1}^{x-N} S_{\tilde{k}}^{*}\left(z_{N+k}+d_{N+k}^{(x-N-k)}(\mathbf{z})\right) \\
& +2 \sum_{j=1}^{N} \hat{\gamma}_{N}^{(j)}\left(z_{1}+d_{1}^{(x-1)}(\mathbf{z}), \ldots, z_{N}+d_{1}^{(x-N)}(\mathbf{z})\right)\left(\mathbb{1}_{\{j \in J\}}+\frac{d}{d z} d_{j}^{(x-j)}(\mathbf{z})\right) \\
& \times \sum_{k=1}^{x-N} \frac{d}{d z} S_{\tilde{k}}^{*}\left(z_{N+k}+d_{N+k}^{(x-N-k)}(\mathbf{z})\right)\left(\mathbb{1}_{\{N+k \in J\}}+\frac{d}{d z} d_{N+k}^{(x-N-k)}(\mathbf{z})\right) \\
& \times \prod_{l \neq k} S_{\tilde{l}}^{*}\left(z_{N+l}+d_{N+l}^{(x-N-l)}(\mathbf{z})\right)+\hat{\gamma}_{N}\left(z_{1}+d_{1}^{(x-1)}(\mathbf{z}), \ldots, z_{N}+d_{N}^{(x-N)}(\mathbf{z})\right) \\
& \times \sum_{j=1}^{x-N}\left[\frac{d^{2}}{d z^{2}} S_{\tilde{j}}^{*}\left(z_{N+j}+d_{N+j}^{(x-N-j)}(\mathbf{z})\right)\left(\mathbb{1}_{\{N+j \in J\}}+\frac{d}{d z} d_{N+j}^{(x-N-j)}(\mathbf{z})\right)^{2} \prod_{k \neq j} S_{\tilde{k}}^{*}\left(z_{N+k}+d_{N+k}^{(x-N-k)}(\mathbf{z})\right)\right. \\
& +\frac{d}{d z} S_{\tilde{j}}^{*}\left(z_{N+j}+d_{N+j}^{(x-N-j)}(\mathbf{z})\right) \frac{d^{2}}{d z^{2}} d_{N+j}^{(x-N-j)}(\mathbf{z}) \prod_{k \neq j} S_{\tilde{k}}^{*}\left(z_{N+k}+d_{N+k}^{(x-N-k)}(\mathbf{z})\right) \\
& +\frac{d}{d z} S_{\tilde{j}}^{*}\left(z_{N+j}+d_{N+j}^{(x-N-j)}(\mathbf{z})\right)\left(\mathbb{1}_{\{N+j \in J\}}+\frac{d}{d z} d_{N+j}^{(x-N-j)}(\mathbf{z})\right) \\
& \left.\times \sum_{k \neq j} \frac{d}{d z} S_{\tilde{k}}^{*}\left(z_{N+k}+d_{N+k}^{(x-N-k)}(\mathbf{z})\right)\left(\mathbb{1}_{\{N+k \in J\}}+\frac{d}{d z} d_{N+k}^{(x-N-k)}(\mathbf{z})\right) \prod_{l \neq k} S_{\tilde{l}}^{*}\left(z_{N+l}+d_{N+l}^{(x-N-l)}(\mathbf{z})\right)\right] \\
&
\end{aligned}
$$

with $\frac{d^{2}}{d z^{2}} d_{j}^{(x-j)}(\mathbf{z})$, being the derivative of (14), recursively given by

$$
\frac{d^{2}}{d z^{2}} d_{j}^{(x-j)}(\mathbf{z})=\sum_{k=j+1}^{N+j}\left[\frac{d^{2}}{d z^{2}} \delta_{\tilde{k}}\left(z_{k}+d_{k}^{(x-k)}(\mathbf{z})\right)\left(\mathbb{1}_{\{k \in J\}}+\frac{d}{d z} d_{k}^{(x-k)}(\mathbf{z})\right)^{2}+\frac{d}{d z} \delta_{\tilde{k}}\left(z_{k}+d_{k}^{(x-k)}(\mathbf{z})\right) \frac{d^{2}}{d z^{2}} d_{k}^{(x-k)}(\mathbf{z})\right] \mathbb{1}_{\{N<k \leq x\}} .
$$

Note that $\frac{d}{d z} d_{j}^{(x-j)}(\mathbf{z})$ is also recursively defined and given in (14).

\section{References}

[1] M.A.A. Boon, R.D. Van der Mei, E.M.M. Winands, Applications of polling systems, Surv. Oper. Res. Manag. Sci. 16 (2) (2011) 67-82.

[2] V.M. Vishnevskii, O.V. Semenova, Mathematical methods to study the polling systems, Autom. Remote Control 67 (2) (2006) 173-220.

[3] O.J. Boxma, M.R.H. Mandjes, O. Kella, On a queuing model with service interruptions, Probab. Engrg. Inform. Sci. 22 (04) (2008) $537-555$

[4] O.J. Boxma, O. Kella, M.R.H. Mandjes, On a generic class of Lévy-driven vacation models, Probab. Engrg. Inform. Sci. 24 (01) (2010) 1-12. 
[5] W. Bux, H.L. Truong, Mean-delay approximation for cyclic-service queueing systems, Perform. Eval. 3 (3) (1983) 187-196.

[6] A.G. Konheim, H. Levy, M.M. Srinivasan, Descendant set: an efficient approach for the analysis of polling systems, IEEE Trans. Commun. 42 (234) (1994) $1245-1253$.

[7] U. Yechiali, Analysis and control of polling systems, in: Performance Evaluation of Computer and Communication Systems, Springer, 1993, pp. 630-650.

[8] O.J. Boxma, J.A. Weststrate, U. Yechiali, A globally gated polling system with server interruptions, and applications to the repairman problem, Probab. Engrg. Inform. Sci. 7 (2) (1993) 187-208.

[9] R.D. Van der Mei, Delay in polling systems with large switch-over times, J. Appl. Probab. 36 (1) (1999) $232-243$.

[10] N.H. Bingham, R.A. Doney, Asymptotic properties of supercritical branching processes I: The Galton-Watson process, Adv. Appl. Probab. (1974) 711-731.

[11] N.H. Bingham, C.M. Goldie, J.L. Teugels, Regular Variation, vol. 27, Cambridge University Press, 1989.

[12] O.J. Boxma, V. Dumas, The busy period in the fluid queue, in: ACM SIGMETRICS Performance Evaluation Review, vol. 26, ACM, 1998, pp. 100-110.

[13] Q. Deng, A two-queue polling model with regularly varying service and/or switchover times, Stoch. Models 19 (4) (2003) 507-526.

[14] W. Feller, An Introduction to Probability Theory and its Applications, Volume II, Wiley, 1971.

[15] H. Takagi, Analysis of Polling Systems, MIT Press, 1986.

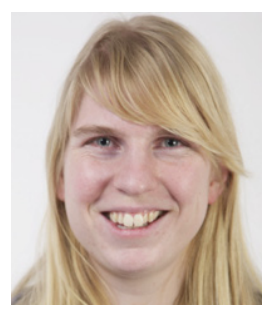

Petra Vis received her M.Sc. degree in Business Analytics in 2012 from the VU University Amsterdam. In 2013 she started as a Ph.D. candidate at the same university. Her topics of interest lie mainly in the field of queueing theory.

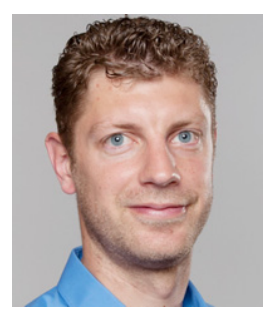

René Bekker received his master's degree from the Econometrics Department of the VU University Amsterdam in 2001 and a Ph.D. from the mathematics department of Eindhoven University of Technology in 2005. Subsequently, he was a post-doc at the CWI (Center for Mathematics and Computer Science) with a long-term visit at INRIA (Sophia Antipolis). In 2006, he joined the Mathematics department of the VU University Amsterdam, where he is an assistant professor in Applied Probability and Operations Research. His primary research interests are in stochastic modeling queueing theory, and performance analysis of service systems, with health care as his favorite application area.

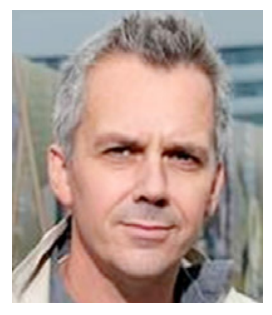

Rob van der Mei is the leader of the research theme Logistics and the Industrial Liaison Officer at CWI, and a full professor at the VU University, Amsterdam. Before going to academia, he has been working for over a decade as a consultant and researcher in the ICT industry, working for PTT, KPN, AT\&T Labs and TNO ICT. His research interests include queueing theory, performance analysis of ICT systems, health logistics, road traffic management, logistics, grid computing, revenue management, military operations research, sensor networks, call centers and data science. He is the co-author of some 130 papers in journals and refereed proceedings. 